\title{
Germanica
}

\section{Sogar Papageien überleben uns d'Olga Martynova. Un exemple de roman interculturel}

Sogar Papageien überleben uns by Olga Martynova. An intercultural novel Sogar Papageien überleben uns von Olga Martynova. Ein interkultureller Roman

\section{Emmanuelle Aurenche-Beau}

\section{OpenEdition}

\section{Journals}

Édition électronique

URL : http://journals.openedition.org/germanica/2029

DOI : 10.4000/germanica.2029

ISSN : 2107-0784

Éditeur

Université de Lille

\section{Édition imprimée}

Date de publication : 31 décembre 2012

Pagination : 191-201

ISBN : 9782913857308

ISSN : 0984-2632

\section{Référence électronique}

Emmanuelle Aurenche-Beau, «Sogar Papageien überleben uns d'Olga Martynova. Un exemple de roman interculturel », Germanica [En ligne], 51 | 2012, mis en ligne le 14 janvier 2013, consulté le 06 octobre 2020. URL : http://journals.openedition.org/germanica/2029 ; DOI : https://doi.org/10.4000/ germanica.2029

Ce document a été généré automatiquement le 6 octobre 2020.

(C) Tous droits réservés 


\title{
Sogar Papageien überleben uns d'Olga Martynova. Un exemple de roman interculturel
}

\author{
Sogar Papageien überleben uns by Olga Martynova. An intercultural novel \\ Sogar Papageien überleben uns von Olga Martynova. Ein interkultureller \\ Roman
}

\section{Emmanuelle Aurenche-Beau}

Sogar Papageien überleben uns ${ }^{1}$ est le premier roman d'olga Martynova, écrivaine russe née en 1962 et venue en Allemagne en 1991, à près de 30 ans. À Leningrad déjà, elle était engagée avec son mari dans les milieux littéraires "alternatifs » : au début des années 1980, ils avaient fondé un groupe appelé Kamera Chranenija (cette expression signifie « consigne » pour indiquer que les samizdats édités par le groupe étaient des lieux de conservation de textes qui n'avaient aucune chance d'être publiés en URSS) ${ }^{2}$. Depuis le début des années 1990, ils étaient également tous deux correspondants de Radio Free Europe/Radio liberty, radios financées par les États-Unis pour combattre le communisme et diffuser des informations inaccessibles aux populations des pays du bloc soviétique ${ }^{3}$. Comme l'explique Alexandra Scherle qui a interviewé Olga Martynova pour la Deutsche Welle, leur décision de s'installer en Allemagne est le fruit d'une « succession de heureux hasards » : en 1991 (à une période de complet chaos en Russie), son mari, Oleg Jurjew et elle sont invités à faire des lectures en Allemagne; son mari se voit proposer un contrat par un éditeur allemand, puis il obtient une bourse et olga Martynova de conclure : « Nous sommes restés parce que nous nous sentions bien ici nous avions la possibilité d'y travailler et d'y vivre - et aussi bien sûr par curiosité ! »4. Si les premiers temps ont peut-être été difficiles du fait qu'elle ne parlait pas allemand, elle semble s'être assez rapidement intégrée dans la vie culturelle de son pays d'accueil, publiant deux recueils de poèmes traduits du russe (essentiellement par elle-même et par Elke Erb ainsi que quelques autres traducteurs), Brief an die Zypressen, paru en 2001 aux éditions Rimbaud et In der Zugluft Europas, paru en 2009 aux éditions Wunderhorn 5 . Depuis la fin des années 1990, elle rédige également régulièrement des critiques 
consacrées à la littérature russe ou d'Europe centrale et orientale du xxe et du xxie siècle pour les journaux allemands les plus prestigieux: Die Zeit, Die Welt, Der Tagesspiegel, Rheinischer Merkur, Süddeutsche Zeitung ${ }^{6} . .$. Des essais croisés, rédigés par son mari et par elle-même, également publiés à l'origine dans la presse, viennent en outre de faire l'objet d'une publication sous le titre Zwischen den Stühlen ${ }^{7}$. Et cette bibliographie déjà impressionnante comprend donc aussi un roman écrit directement en allemand qui a obtenu le "Förderpreis zum Albert von Chamisso-Preis » (prix récompensant une œuvre écrite en allemand par un auteur dont l'allemand n'est pas la langue maternelle).

2 Son personnage principal, Marina, qui est en même temps la narratrice, est une jeune Russe de 36 ans qui enseigne la littérature allemande à l'Université de SaintPétersbourg et qui vient en Allemagne faire une tournée de conférences sur les obérioutes, un groupe d'écrivains russes d'avant-garde de l'entre-deux-guerres, tombés en disgrâce pendant la période soviétique, mais dont les œuvres circulaient tout de même sous le manteau, en particulier dans les milieux oppositionnels des années 1970-1980 ${ }^{8}$. Marina est en particulier invitée à un festival de littérature dont le but est de faire connaître, par des lectures et des conférences, la littérature russe à un public allemand .

3 Ce premier niveau du roman qui se situe en 2006 sert de cadre temporel à plusieurs retours en arrière clairement situés chronologiquement grâce à une échelle temporelle qui ouvre chacun des chapitres du livre: les années correspondant aux événements évoqués dans le chapitre sont en gras. En dehors de l'année 2006, une autre année-clé du roman est l'année 1986, l'année où Marina fait la connaissance d'un étudiant allemand en slavistique, Andreas, venu passer un semestre à Leningrad et dont elle tombe amoureuse. Les autres moments qu'ils passent ensemble entre 1986 et 2006 leur voyage en Asie centrale en 1988, leur séjour à Rome en 2002 et leurs diverses rencontres à Berlin ou à Leningrad - font également l'objet de chapitres spécifiques. Malgré les difficultés liées au contexte politique, surtout jusqu'à la fin de l'URSS, Marina et Andreas ne se sont donc pas perdus de vue au cours de ces vingt années. Mais ils se sont pour ainsi dire continuellement manqués : en 1990, Andreas a épousé une Allemande et Marina, quelque temps après, s'est mariée elle aussi, mais l'un et l'autre ont divorcé et au bout de toutes ces années de malentendus, Andreas vient de faire à Marina une demande en mariage. Le fils conducteur du roman est constitué par l'évocation des souvenirs et des ambivalences de Marina, partagée entre peur et envie de répondre positivement à Andreas et de venir s'installer à Berlin.

4 Le roman ne se réduit cependant pas à la chronique de cette histoire d'amour, il propose aussi un tableau assez surprenant du monde disparu qu'est la Russie soviétique auquel nous consacrerons notre première partie. Nous étudierons ensuite la manière dont Olga Martynova relit certains chapitres de l'histoire des relations germanorusses/soviétiques et conclurons, après une réflexion sur l'écriture du roman, en tentant de montrer de quelle manière le livre s'inscrit dans une littérature interculturelle.

5 Si le cadre du roman est constitué, comme on l'a vu, par le récit du séjour effectué par la narratrice en Allemagne à l'occasion du festival de littérature auquel elle a été invitée, une bonne partie des chapitres du roman est consacrée à une évocation des dernières années de l'Union Soviétique (de 1986 au putsch d'août 1991 ${ }^{10}$ ). On y trouve tout d'abord quelques aspects attendus: le manque de certaines denrées - dans un 
inventaire à la Prévert, la narratrice énumère ainsi un certain nombre de « choses de l'autre vie " ${ }^{11}$, c'est-à-dire d'objets de l'Ouest introuvables en URSS : " une bouteille de gin vidée depuis longtemps avec une branche de genévrier sur l'étiquette $»^{12}$, « un magazine de mode, disons Vogue » qui «posé à proximité du programme de télévision au papier soviétique jaunâtre a l'air d'un papillon tropical à côté de mouches indigènes ${ }^{13}$, une boîte de chocolat en fer blanc au décor Jugendstil, et sa « doudoune ", un cadeau d'Andreas (SP, 16) ${ }^{14}$. Elle fait aussi allusion aux activités de la Sécurité d'État, mais surtout pour souligner que sa surveillance s'est assouplie et que les relations avec les étrangers sont désormais tolérées ${ }^{15}$. Les frontières sont également devenues moins infranchissables et elle peut ainsi rendre visite à Andreas en Allemagne ${ }^{16}$.

6 Le côté le plus étonnant de cette évocation des dernières années de la Russie réside cependant dans le portrait qu'elle brosse d'un milieu d'artistes et d'originaux qui forment une sorte de microcosme vivant en marge de la société soviétique, cultivant, grâce aux « objets d'avant $~^{17}$ qu'elle oppose aux « objets de la vie soviétique $»^{18}$, le lien avec le monde d'avant la Révolution ${ }^{19}$ et maintenant la tradition d'une littérature interdite par le pouvoir en place, notamment celle des Obérioutes - elle se voit ainsi offrir à plusieurs reprises des copies de manuscrits de Daniil Harms, un de ses principaux représentants ${ }^{20}$. Marina évoque en outre l'existence de communautés hippies vivant loin des villes et de la réalité soviétique. Elle cite notamment l'exemple d'un couple d'artistes moscovites, devenus apiculteurs en Asie centrale et semblant être au cœur d'un vaste réseau de contacts et d'adresses dans l'ensemble du pays ${ }^{21}$. Elle leur rend visite au cours d'un voyage rocambolesque en compagnie du poète underground Fjodor, de son ami allemand Andreas et d'un étudiant américain, John et commente ainsi les paradoxes de la Russie de la fin des années 1980 : «Il y avait à l'époque une coexistence étrange entre liberté et absence de liberté ${ }^{22}$. Elle évoque aussi un couple d'amis étudiant l'hiver à Moscou et passant l'été à parcourir l'Asie centrale en se présentant auprès des autorités comme collaborateurs d'une expédition à la recherche d'un homme des neiges (yéti), reconnaissant elle-même l'aspect « incroyable » de cette réalité ; elle explique par la suite que ce genre d'expédition est à resituer dans le contexte de la guerre froide et de la concurrence à laquelle se livraient Russes et Américains, aussi bien dans le domaine de la conquête spatiale que dans celui de la capture du premier homme des neiges!

7 Un autre phénomène peu connu et assez inattendu évoqué dans le roman est, à l'occasion d'un voyage de Marina en Bouriatie (région de Sibérie orientale proche du lac Baïkal) ${ }^{23}$ qui occupe une bonne partie de la sixième partie du roman, celui de la survivance de formes de présence religieuse: rites chamaniques, monastères bouddhistes officiels et non officiels ayant résisté à la propagande athéiste du régime ${ }^{24}$. Marina s'intéresse particulièrement à l'histoire du "lama imputrescible», censé "revenir vivant», dont le cercueil en bois de pin se trouve dans un lieu tenu secret dans lequel elle se rend avec ses amis et où elle se voit, à son grand étonnement, déposer une offrande (" une offrande ? pour qui ?», SP 156).

8 Si le roman offre donc une image à la fois assez partielle (puisqu'elle se limite à l'évocation d'un milieu oppositionnel) et assez surprenante des dernières années de l'Union soviétique, l'histoire d'amour de Marina et d'Andreas et leurs allers et retours entre la Russie et l'Allemagne permettent d'aborder quelques aspects des relations entre les deux pays. Le roman insiste notamment beaucoup sur la présence de l'Histoire, en particulier sur les souvenirs liés à la période de la Seconde Guerre 
mondiale qui hante encore très fortement les esprits, aussi bien côté allemand que côté russe. Quand le père d'Andreas apprend que son fils a décidé de faire des études de russe et qu'il a en outre l'intention de partir étudier un semestre dans une université soviétique, sa première réaction, à lui qui a perdu son bras droit lors du siège de Leningrad, est de "se taire», de "se taire pendant une semaine entière » (SP 27). Marina décrit, de même, le « choc » de ses parents quand elle leur annonce la visite de son petit ami allemand (SP 26). Elle évoque en outre des discussions qu'elle a avec des collègues allemands pendant le festival de littérature auquel elle participe en 2006. Elle raconte notamment comment elle est interpelée par une poétesse allemande qui ne comprend pas qu'on puisse écrire dans une langue de bourreaux et de violeurs (la langue russe); ses tentatives de lui faire comprendre que les exactions commises par les Allemands sont sans commune mesure avec celles commises par les Russes semblent la laisser de marbre (SP 113-114). Outre l'extermination systématique des Juifs que lui rappelle Marina, le roman évoque à plusieurs reprises le souvenir du siège de Léningrad $^{25}$ : la grand-mère de son amie Antonia confirme par exemple que la famine était telle qu'on a réellement mangé du chat (SP 126), ajoutant que son oncle avait ensuite conservé la peau des chats dans l'espoir de l'échanger au marché noir contre du pain (SP 127) ; un des chapitres du roman rappelle aussi les conditions de la mort de l'écrivain Daniil Harms, mort de faim en 1942 dans la prison où il était incarcéré pour activités subversives, alors que sa femme tentait de lui apporter un peu de pain, «cela signifiait, dans la Léningrad assiégée, qu'elle lui apportait sa propre ration, alors qu'elle était elle-même près de mourir d'inanition $»^{26}$.

Une des conséquences de ce lourd passé de souffrances est la question qui revient à plusieurs reprises dans le livre d'une sorte de retour à la normalité que les uns et les autres ont du mal à reconnaître à leurs anciens ennemis. Marina supporte ainsi difficilement les manifestations de fierté nationale allemande retrouvée à l'occasion de la coupe du monde de football : l'omniprésence du drapeau allemand, les supporters dans les rues, les télévisions allumées dans tous les cafés... Elle se montre par ailleurs assez sceptique quand elle entend le propriétaire de la demeure où a lieu le festival évoquer les anciens propriétaires juifs et se présenter comme un fin connaisseur des coutumes juives, se disant qu'elle pourrait lui demander s'ils ont pu partir à temps ${ }^{27}$. Elle s'interroge aussi sur la présence d'une xénophobie latente dans l'Allemagne contemporaine. Son attention est attirée par exemple par une inscription sur un mur : «Kanaken, Koffer packen!» qui témoigne d'un racisme anti-turc. Et alors qu'elle rapporte une scène où elle voit plusieurs jeunes Allemands se montrer solidaires d'un jeune Noir subissant un contrôle de titre de transport (et se révélant finalement être un fraudeur), c'est-à-dire, pour reprendre les mots de l'écrivain russe Nikolaj qui assiste également à la scène, "se montrer prêts à prendre la défense de minorités ${ }^{28}$, elle la conclut par les mots de son ami Fjodor pour qui cette scène semble, au contraire, la preuve que le racisme n'a pas disparu: "C'est un paradoxe, si ces bonnes personnes n'étaient pas racistes au plus profond d'elles-mêmes, elles auraient simplement passé leur chemin, nous aussi bien sûr. Vous comprenez : ou bien nous sommes racistes et la couleur de la peau des uns et des autres nous importe, ou bien nous ne le sommes pas et cela nous est égal, et nous ne nous serions jamais retrouvés dans une situation aussi ridicule $»^{29}$.

10 Face à ces réactions russes vis-à-vis des réalités allemandes, on peut citer la réaction d'une Allemande, présidente d'une association germano-russe coorganisatrice du festival, à l'égard des transformations de la Russie: de retour d'un voyage à Saint- 
Petersbourg à l'occasion du 300e anniversaire de la ville en 2003, elle affirme sur un ton assez péremptoire que tous ces changements ne sont que de la poudre aux yeux et emploie l'expression « village de Potemkine » qui fait vivement réagir Marina qui tente de lui expliquer la véritable origine de cette expression, inventée en réalité... par l'ambassadeur de Saxe (SP 132-133).

11 Mais par-delà ces quelques notations qui montrent la complexité des relations entre Allemands et Russes, le roman est aussi une interrogation sur la manière de dire cet entre-deux pays/cultures. Un des principaux moyens auquel a recours Olga Martynova semble être celui d'un jeu intertextuel qui fonctionne à plusieurs niveaux.

On trouve tout d'abord dans le roman des allusions explicites à des auteurs aussi bien de langue allemande que de langue russe qui, par-delà leur diversité apparente, semblent renvoyer à un courant littéraire que l'on pourrait subsumer sous une notion de non-sens ou d'absurde qui, paradoxalement, pourrait être pour Olga Martynova la mieux à même de donner à comprendre cette situation d'entre-deux. Cette notion est présente de manière tout à fait explicite avec les allusions à « Harms et ses amis qui ont choisi le non-sens comme moyen de connaissance", pour "comprendre le monde absurde dans lequel ils avaient à vivre $»^{30}$, à Tchékhov " dont les histoires du quotidien annoncent le théâtre absurde $\aleph^{31}$ ou à Thomas Bernhard et à sa merveilleuse capacité à inventer des « équations absurdes » et à les présenter comme des vérités allant de soi ${ }^{32}$. Cette notion est aussi introduite dans le roman sous la forme de vignettes textuelles comme l'allusion à la scène du roman Liebesblödigkeit de Wilhelm Genazino (2005) où l'on voit un personnage abandonner une valise sur une place de ville et attendre de voir ce qu'il en advient (SP 10) ou au texte de Gombrowicz dans lequel le narrateur raconte que c'est dans le Tiergarten de Berlin qu'il a fait l'expérience directe de la mort sous la forme d'un oiseau invisible perché sur son épaule et ne le quittant plus (SP 39). Surtout, le titre du roman lui-même, Sogar Papageien überleben uns, une citation à première vue assez énigmatique de Joseph Roth, apparaît comme une parfaite illustration de cette conception de la littérature, dans la mesure où il semble illustrer cette idée qu'il est impossible de rendre compte du monde (absurde) qui nous entoure, sinon par une écriture de l'absurde. La citation de Vvédenski, un des poètes obérioutes proche de Harms, qui clôt le roman l'indique assez clairement :

« Toute description est fausse. La phrase : 'Un homme est assis, sur sa tête il y a un

bateau' est plus juste que 'Un homme est assis et lit un livre' » (SP 199).

13 La phrase de Roth est néanmoins présente à l'intérieur du roman dans un chapitre intitulé « chiromancie » (« Handlesen »). Marina y évoque une scène de l'année 2005; elle se trouvait à Berlin au café Joseph Roth dans la Potsdamer Strasse, en compagnie d'Andreas et d'un artiste allemand, Gregor, à qui elle devait apporter les catalogues d'un artiste russe : " Je pris un des livres de Joseph Roth sur l'étagère et le feuilletai. Je lus à haute voix : '...Même les perroquets nous survivent.' $»^{33}$. S'ensuit une discussion sur les diseuses de bonne aventure, Gregor tire de sa poche une prédiction qu'il vient de sortir d'un distributeur de luna-park où il était juste auparavant avec sa fille. Andreas se montre très sceptique, Marina s'en amuse et se met à lire les lignes de la main de Gregor, puis elle lui montre les siennes, notamment sa ligne de vie en lui faisant remarquer qu'elle s'interrompt pour repartir plus loin. Or cette scène qui traite d'un sujet à première vue peu rationnel et peu sérieux et qui peut sembler gratuite et anecdotique touche en réalité au cœur de la problématique du roman. Marina conclut par ces mots : 
"J'aurais pu dire à Andreas: C'est comme avec nous. Tu sais, cette interruption de ma ligne de vie peut même effectivement signifier ça-je veux dire, que tu m'as quittée à l'époque et que maintenant tu ne me laisses pas en paix. Mais je dis : 'Et les rouges-gorges ne vivent qu'un an, en moyenne. C'est ce qu'on dit. Je l'ai lu'. » ${ }^{34}$. roman : la narratrice s'y voit au milieu d'un torrent dans les bras d'une femme qui ne bouge pas; pourtant, au bout d'un moment, elle se voit sur l'autre rive, sans comprendre comment cela a pu se produire (SP 7) ${ }^{35}$ ! On peut en effet se demander si cette scène étrange ne contient pas pour ainsi dire le programme du roman. Le roman lui-même n'est-il pas précisément le lieu d'un passage, du passage de la Russie à l'Allemagne par le biais de la transposition par une écrivaine russe dans une œuvre écrite directement en allemand d'un pan important, mais méconnu de la littérature russe du xxe siècle, la littérature des Obérioutes? On y retrouve en tout cas un certain nombre des procédés d'écriture caractéristiques de Harms et de son cercle $^{36}$. À commencer par cette propension aux textes courts centrés sur un fait, sur une situation souvent loufoque ou cocasse et qui constituent à eux seuls un (bref) chapitre du roman - les exemples sont nombreux en dehors de la scène de café précédemment citée, on peut citer la scène de la brosse à dents électrique qui vibre dans son sac au moment où Marina passe la douane et provoque la panique des douaniers (SP 9) ou encore celle où Marina et ses amis sont sauvés par la montre de Fjodor qui porte l'inscription « 50 ans de la police des Chemins de fer »: lorsque Fjodor dit aux miliciens qu'elle vient de son père, alors qu'il l'a en réalité reçue en cadeau d'un ami, ils sont libérés sans même que leur identité soit contrôlée (SP 63-64)!

Un autre trait de l'écriture d'Olga Martynova qui pourrait être emprunté aux Obérioutes est la présence de chapitres constitués de dialogues semblant directement retranscrits à partir de scènes réelles - elle évoque à plusieurs reprises la technique "photographique» de transcription des conversations pratiquée notamment par le poète obérioute Lipavski qui s'était mis à "prendre en notes leurs conversations, il appelait cela les 'photographier' " ${ }^{37}$ (SP 52). Là aussi les exemples sont nombreux, entre les conversations avec les autres participants au festival - citons la discussion du petitdéjeuner où les uns et les autres débattent du nom des oiseaux qu'ils ont entendu chanter pendant la nuit (rossignols pour les Allemands, alouettes, merles ?) (SP 35-36), la retranscription de réflexions de jeunes femmes à propos des relations hommesfemmes entendues pendant le cocktail qui suit sa conférence (SP 81) ou encore les conversations déjà évoquées avec la poétesse allemande (SP 112-113) ou avec la présidente de l'association germano-russe (SP 132-133).

D'autres chapitres du roman, enfin, sont constitués d'échantillons de textes du poète underground Fjodor lus pendant le festival. Ils frappent par leur écriture extrêmement sèche et dépouillée et par la maîtrise des chutes qui rappellent très directement les textes de $\mathrm{Harms}^{38}$. Chacun d'eux semble en outre condenser en quelques lignes ce qui fait le cœur du roman, c'est-à-dire la question de l'entre-deux, de la possibilité ou de l'impossibilité de choisir entre deux vies, de choisir de changer de vie. On peut citer, par exemple, le texte intitulé "Naissance d'une danseuse» qui évoque Mata Hari mourante et un papillon qui attend de savoir ce qu'elle voudrait être dans une prochaine vie : «Une danseuse, pensa Matilda Haritonovna » ${ }^{39}$ ou ceux intitulés « De la vie d'un pharmacien (I) » et «De la vie d'un pharmacien (II)»: dans le premier, est évoqué le jour où il prendra conscience de tout ce qu'il ne peut plus faire «jusqu'à ce qu'il ne reste plus qu'une possibilité... », dans le deuxième, le jour de sa rencontre avec 
une jeune Indienne dont il tombera amoureux, « comme si ces vingt années n'avaient pas existé, comme si tu n'étais pas encore devenu pharmacien... $»^{40}$. On peut penser aussi aux «Réflexions d'une mouche » qui a deux miroirs et qui n'arrive pas à savoir dans lequel elle se trouve la plus belle : «C'est bien pour ça que j'ai les deux $»^{41}$.

Ce qui fait la force du roman est donc cette attention égale portée à chacun des chapitres (chacun d'eux pourrait être lu de manière autonome) et au lien de chacun des chapitres à ce qui fait le fil conducteur du roman, l'histoire d'amour entre Marina et Andreas, le choix de Marina entre la Russie et l'Allemagne. Le roman d'olga Martynova occupe en outre une place originale dans le vaste champ de la littérature interculturelle. Elle crée en effet une œuvre hybride par sa manière de reprendre en allemand des pratiques d'écriture typiques d'une certaine littérature russe méconnue, mais essentielle pour l'histoire de la littérature - dans un de ses essais, elle qualifie les Obérioutes de « derniers représentants de la modernité russe » avant "la longue nuit soviétique $»^{42}$. À la différence d'autres auteurs qui jouent davantage sur des pratiques langagières de créolisation, Olga Martynova pratique une hybridité intertextuelle que l'on pourrait rapprocher de celle d'auteurs comme l'écrivain d'origine syrienne Rafik Schami qui reprend dans ses romans des pratiques d'écriture typiquement orientales comme le conte ou la fable. Pour reprendre les mots de Christoph Jurgensen: "Les textes vont et viennent donc aussi bien d'un point de vue thématique que d'un point de vue esthétique entre les littératures / entre les cultures et cherchent ainsi leur place, leur positionnement esthétique dans l'entre-deux en proposant ainsi au lecteur au sens métaphorique du terme des "voyages" dans l'espace intermédiaire entre les langues et les cultures $»^{43}$.

\section{NOTES}

1. Droschl, Graz, Wien, 2010. Les citations tirées du roman seront données en utilisant l'abréviation SP suivie du numéro de la page.

2. Cf. Roman Widder, «Ein Dialog zwischen den Sprachen. Ein Porträt der Lyrikerin Olga Martynova ", http://www.novinki.de/html/vorgestellt/Portrait_Martynova.html consulté le 30-5-2012. Les activités du groupe se prolongent jusqu'à aujourd'hui sous la forme d'un site internet : http://newkamera.de/ojuriew.html consulté le 30-5-2012.

3. Fondées dans les années 50, elles ont fusionné en 1976 ; leur siège qui était initialement à Munich a déménagé à Prague en 1995. On trouve sur leur site la définition précise de leurs buts : cf. http://www.rferl.org/info/mission/169.html consulté le 30-5-2012. Ajoutons que ces radios offraient aussi des possibilités de travail à un certain nombre d'écrivains émigrés (cf. Elena Tichomirova, «Literatur der russischen Emigrant/innen », in Carmine Chiellino, Interkulturelle Literatur in Deutschland, Metzler, Stuttgart, 2007, p. 169).

4. « Weil wir uns hier sehr wohl gefühlt haben - und die Möglichkeit hatten, hier zu arbeiten und zu leben - sind wir geblieben. Und natürlich aus Neugier ", "Deutschland ist mein Zuhause » Deutsche Welle 21-3-11.

5. Pour une présentation de son œuvre lyrique on pourra se reporter au texte de Roman Widder «Ein Dialog zwischen den Sprachen. Ein Porträt der Lyrikerin Olga Martynova » (cf. note 1). 
6. Un choix de ces critiques a été publié en 2003 par les éditions Rimbaud sous le titre Wer schenkt was wem.

7. Bernstein, 2011.

8. Un choix de leurs textes a été publié en français par Macha Zonina et Jean-Christophe Bailly sous le titre Des hommes sont sortis de chez eux, Christian Bourgois, Paris, 1997.

9. Un certain nombre de ses poèmes ayant été traduit en 2003 lors de l'atelier de traduction de poésie de la Maison des artistes de Edenkoben (Rhénanie-Palatinat), comme l'indique la postface à son recueil In der Zugluft Europas, on peut se demander si elle ne s'est pas inspirée de cette expérience dans son roman. Précisons qu'Olga Martynova a également été en 2007 boursière de cette même institution (après Elke Erb en 2002). http://www.kuenstlerhaus-edenkoben.de/203/ consulté le 30-5-2012.

10. Rappelons que l'année 1991 est aussi l'année où Olga Martynova quitte son pays d'origine.

11. « Dinge aus dem anderen Leben » (SP 15).

12. « Eine längst ausgetrunkene Flasche Gin, ein Wacholderzweig auf ihrem Etikett », (SP 16).

13. "Eine Modezeitschrift, sagen wir Vogue. Auf einem Beistelltischchen liegt sie neben dem Fernsehprogramm mit seinem sowjetisch-gelblichen Papier wie ein tropischer Schmetterling neben einheimischen Fliegen. » (SP 16). Cf. aussi SP 151 où l'on trouve une autre énumération de denrées introuvables.

14. Elle rappelle également la pénurie qui régnait dans les dernières années du régime et le retour des tickets de rationnement: «Die Lebensmittel wurden knapp. Man bekam Karten für den Kauf einer bestimmten Menge pro Monat von Zucker, Butter, Waschpulver» (SP 48) et d'énumérer tout ce qu'Andreas sortait de son sac à dos quand il arrivait : « Andreas stellte seinen Rucksack auf den Küchentisch und holte Schokolade, Kekse, Wurst und viele andere gute Sachen hervor, die es, hatte er gehört, bei uns nicht mehr gab. » (SP 49).

15. «Das ausgehende Jahrhundert war zu einem fetten faulen Köder geworden. Kaum etwas an ihm erinnerte an jenen Wolfshund, dessen wahnsinnige Augen Mandelstam um Verstand und Leben gebracht hatten. Ausländische Freunde (Personen aus dem anderen Leben) zu haben galt immer noch als gefährlich, aber die staatliche Sicherheit zeigte in der Zeit, in der Andreas in Petersburg studierte, kein Interesse an uns. » (SP 23).

16. «Die Zeit begann zu knicken und zu knacken. Die Grenzen wurden locker und ich plante meine erste Reise nach Deutschland. » (SP 48).

17. « Dinge von früher » (SP 13).

18. « Dinge des sowjetischen Lebens » (SP 15).

19. On trouve dans le roman comme exemple de ces "objets d'avant »: un vase rococo indigo, une photo de la belle époque, une peau d'ours, un miroir au cadre d'acajou (SP 13).

20. C'est par l'intermédiaire de sa grand-mère, farouche opposante au régime soviétique qu'elle a été introduite dans cette " société ».

21. «Bald gehörte die Imkerei zum über das ganze Land ausgebreiteten Netz von Kontakten und Adressen, einem Gemeingut bestimmter Künstlerkreise, der Anhänger der sich ausdünnenden Hippiebewegung und aller, die in diesen Freiräumen der Unfreiheit zu sein glaubten. » (SP 68).

22. «Es gab damals ein seltsames Wechselspiel zwischen Freiheit und Unfreiheit. » (SP 68).

23. Après qu'Andreas lui a annoncé son mariage, Marina traverse en effet une grave crise et décide de partir et de se rendre dans un monastère bouddhiste de Bouriatie dont elle a entendu parler par le réseau hippie dont il a déjà été question (SP 149-150).

24. Dans les années 1930, en effet, Staline avait fait arrêter et interner de nombreux religieux, tout en tolérant de nouveau leur présence après la Seconde guerre mondiale pour les remercier d'avoir prié pour la victoire (SP 155).

25. Rappelons que ce siège a duré 872 jours (du 8 septembre 1941 au 27 janvier 1944) et qu'il a fait plus d'un million de victimes civiles. 
26. « das bedeutete im belagerten Leningrad, sie brachte ihm ihre eigene Ration und war selbst nahe am Verhungern. » (SP 37).

27. «Das Landhaus hier hatte vor hundert Jahren eine wohlhabende jüdische Familie errichten lassen, hat mir der jetzige Besitzer erzählt, mit dem Stolz, den ältere deutsche Herren ausstrahlen, wenn sie über Juden mit Sympathie reden, insbesondere, wenn sie über jüdische Bräuche Bescheid wissen (Sehen Sie, liebe Frau Alexandrova, diese Gartenlaube hier war speziell für das Laubhüttenfest gebaut. Wissen Sie, was Laubhüttenfest ist?)... Vielleicht frage ich den Hausherrn nach dem Abendessen, ob sie rechtzeitig haben wegkommen können. » (SP 57).

28. «bereit, Minderheiten zu verteidigen » (SP 174).

29. «Das ist ein Paradoxon: wären diese guten Menschen im Inneren ihres Herzens nicht rassistisch, wären sie einfach vorbeigegangen, wir natürlich auch. Versteht ihr : entweder wir sind Rassisten und es ist uns nicht egal, wer wie gefärbt ist, oder wir sind es nicht, und es ist uns egal, und wir wären in so eine dumme Situation nie geraten. » (SP 174).

30. «Charms und seine Freunde haben den Unsinn als Erkenntnismittel gewählt. Sie wollten die absurde Welt verstehen, in der sie zu leben hatten. » (SP 53).

31. "Tschechow, dessen Alltagsgeschichten schon auf der Schwelle zum absurden Theater stehen » (SP 53).

32. «... Thomas Bernhard wusste wunderbar absurde Gleichungen zu erfinden, die er aber von sich gab mit der Selbstverständlichkeit eines Tolstoi » (SP 32).

33. «Ich nahm eins von Joseph Roths Büchern aus dem Regal und blätterte darin. Ich las vor : '... Sogar Papageien überleben uns'. » (SP 95).

34. «Ich hätte Andreas sagen können : Das ist wie mit uns. Und weisst du, diese Unterbrechung meiner Lebenslinie kann sogar tatsächlich das bedeuten - ich meine, dass du mich damals verlassen hast und nun nicht in Ruhe lässt. Statt dessen sagte ich : 'Und Rotkehlchen leben nur ein Jahr, durchschnittlich. Sagt man. Ich habe das gelesen.' » (SP 97).

35. Cette scène n'est pas sans rappeler un passage de l'autobiographie de Canetti, Die gerettete Zunge, dans lequel il raconte que les contes qu'il a entendus enfant en bulgare se sont mystérieusement traduits en lui en allemand.

36. On peut consulter en français les Écrits de Daniil Harms publiés et traduits par Jean-Philippe Jaccard, Christian Bourgois, 1993 ou ses Euvres en prose et en vers publiées et traduites par Ivan Mignot, Verdier, 2005.

37. « wie... Lenonid Lipawskij dazu übergangen war, ihre Gespräche zu protokollieren, er nannte es 'fotografieren'» (SP 52).

38. La biographie de Fjodor présente en outre un certain nombre de parallèles avec celle de Harms - tous deux refusent de faire des compromis avec le régime soviétique qui les persécute l'appartement de Fjodor est plusieurs fois fouillé, certains de ses manuscrits sont confisqués, il finit comme Harms par ne plus écrire de poésie et par ne plus écrire que de la prose (SP 109). Pour la biographie de Harms, cf. Jean Philippe Jaccard, op. cit., p. 7-21 et 569-574.

39. « Eine Tänzerin, dachte Mathilde Haritonowna. » (SP 85).

40. «bis nur eine Möglichkeit bleibt »; «als wären diese zwanzig Jahre nicht gewesen, als wärst du noch nicht Apotheker geworden... » (SP 146-7).

41. « Na, darum habe ich die zwei. » (SP 108).

42. «Das Leben hat über den Tod gesiegt, auf eine mir unbekannte Reise. Über die lebendigsten aller russischen Klassiker », Zwischen den Stühlen, Bernstein, Bonn, 2011, p. 85-86.

43. «Die Texte bewegen sich folglich sowohl thematisch als auch ästhetisch zwischen den Literaturen bzw. Kulturen hin und her und suchen dabei ihren je eigenen Ort, ihren je eigenen ästhetischen Standpunkt im Dazwischen - und präsentieren dem Leser somit im metaphorischen Sinn 'Reisen', die im Zwischenraum der Sprachen und Kulturen unternommen werden.», «Zwischen den Sprachen, zwischen den Kulturen - Sprach-'Reisen' in der neueren deutschen Literatur », in Patricia Desroches-Viallet, Construction de l'identité dans la rencontre des cultures chez 
les auteurs d'expression allemande. Le voyage immobile, Publications de l'Université de Saint-Étienne, 2009, p. 137.

\section{RÉSUMÉS}

Le roman d'Olga Martynova, écrivaine russe née en 1962 et venue en Allemagne en 1991, relate le séjour en Allemagne de son personnage principal, Marina, universitaire russe participant en 2006 à un festival de littérature dont le but est de faire connaître la littérature russe à un public allemand. En lien avec la relation amoureuse qui unit Marina et Andreas, un étudiant en slavistique que Marina a connu vingt ans plus tôt lorsqu'il était étudiant à Leningrad, le roman brosse un tableau assez surprenant de la fin de la Russie soviétique. Il aborde également la question des relations germano-russes/soviétiques en se centrant principalement sur la période de la Seconde Guerre mondiale et surtout se caractérise par une écriture hybride fondée sur un jeu intertextuel avec l'œuvre de différents écrivains pratiquant une écriture du non-sens, notamment les Obérioutes, un groupe de poètes russes d'avant-garde de l'entre-deux-guerres, à qui est consacrée la conférence de Marina lors du festival de littérature et à qui Olga Martynova emprunte différents procédés d'écriture.

Olga Martynova is a Russian writer, who was born in 1962 and came to Germany in 1991. Her novel relates the stay in Germany of her main protagonist, a Russian academic who takes part in a literature festival whose purpose is to inform a German audience about the Russian literature. In connection with the love story between Marina and Andreas, a young German student of slavistics she met 20 years earlier while he was studying in Leningrad, the novel describes the collapse of the Soviet Union. The novel also examines the question of the German-Russian/Soviet relations, particularly the period of the Second world war. Above all, it is characterized by a hybrid writing that is based on an intertextual play with the works of several writers who practise a nonsense literature, especially the Oberiuts, a group of avant-garde Russian poets of the interwar period to whom Marina devotes her lecture and from whom Olga Martynova borrows several writing processes.

Der Roman Olga Martynovas, einer russischen Schriftstellerin, die 1962 geboren wurde und 1991 nach Deutschland kam, schildert den Aufenthalt seiner Hauptfigur Marina in Deutschland Marina ist eine russische Akademikerin, die an einem Literaturfestival teilnimmt, dessen Ziel darin besteht, einem deutschen Publikum die russische Literatur bekannt zu machen. In Verbindung mit der Liebesbeziehung zwischen Marina und Andreas, einem deutschen Slawistikstudenten, den Marina 20 Jahre früher während seines Studiums in Leningrad kennengelernt hat, gibt uns der Roman ein (überraschendes) Bild der letzten Jahre des sowjetischen Russlands. Der Roman setzt sich auch mit der Frage der deutsch-russichen/ sowjetischen Beziehungen auseinander, wobei er sich vor allem auf die Periode des Zweiten Weltkriegs konzentriert. Er charakterisiert sich aber vor allem durch ein hybrides Schreiben, das auf einem intertextuellen Spiel mit dem Werk verschiedener Autoren beruht, die eine Literatur des Unsinns praktizieren, vor allem der Oberiuten, einer Gruppe avantgardistischer russischer Dichter aus der Zeit zwischen den beiden Weltkriegen, der Marinas Festival-Vortrag gewidmet ist und der Olga Martynova mehrere Schreibverfahren entlehnt. 
INDEX

oeuvrecitee Sogar Papageien überleben uns

\section{AUTEURS}

EMMANUELLE AURENCHE-BEAU

Université Lumière Lyon 2 